\title{
Supercapacitor-battery hybrid energy storage devices from an aqueous nitroxide radical active material
}

\author{
GUO Wei ${ }^{1,2,3}$, XIN Sen ${ }^{1,2,3}$, JI MengBo ${ }^{1,2}$, GUO YuGuo ${ }^{1,2 *} \&$ WAN LiJun ${ }^{1,2}$ \\ ${ }^{1}$ Key Laboratory of Molecular Nanostructure and Nanotechnology, Institute of Chemistry, Chinese Academy of Sciences, Beijing 100190, China; \\ ${ }^{2}$ Beijing National Laboratory for Molecular Sciences, Beijing 100190, China; \\ ${ }^{3}$ Graduate University of Chinese Academy of Sciences, Beijing 100064, China
}

Received August 30, 2010; accepted October 23, 2010

\begin{abstract}
A hybrid electrochemical energy storage device was fabricated in aqueous $\mathrm{NaOH}$ with the 2,2,6,6-tetramethyl-1-piperidinyloxy (TEMPO) nitroxide radical as the active material, hydroquinone as the counter electrode active material, and an $\mathrm{OH}^{-}$-selective separating membrane. The working principle of this device was investigated and it can be considered as a supercapacitor-battery hybrid energy storage system. Device performance was characterized by cyclic voltammetry and galvanostatic charge-discharge testing. When using multi-walled carbon nanotubes (MWCNTs) as electrode support materials, a high pseudo-capacitance of $1280 \mathrm{Fg}^{-1}$ was obtained with the TEMPO nitroxide radical as the active material at a $1 \mathrm{mV} \mathrm{s}^{-1}$ scan rate. This was $\sim 33$ times larger than the inherent double layer capacitance of MWCNTs. The electrode material and active material dissolved in solution could potentially be substituted with similar materials. This simple design provides a new approach for fabricating high performance supercapacitor-battery hybrid energy storage devices.
\end{abstract}

nitroxide radical, electrochemical energy storage device, hybrid system, reversible redox reaction, aqueous solution electrolyte

Citation: Guo W, Xin S, Ji M B, et al. Supercapacitor-battery hybrid energy storage devices from an aqueous nitroxide radical active material. Chinese Sci Bull, 2011, 56: 2433-2436, doi: 10.1007/s11434-011-4575-Z

The demand for advanced electrochemical energy storage devices with increased power and energy densities is continuously increasing because of sustainable energy and environmental issues $[1,2]$. Supercapacitors represent the state of the art in high power systems, while rechargeable batteries (particularly lithium-ion batteries) are the highest energy storage facilities [3-5]. Hybrid energy storage devices, using both supercapacitor and battery electrodes in a single unit cell, provide a clever solution combining the advantages of the two energy storage systems. Clearly, such high performance hybrid devices cannot necessarily be achieved by combining any supercapacitor and rechargeable battery. Supercapacitors and rechargeable batteries operate over different potential ranges and in different electrolytes

*Corresponding author (email: ygguo@iccas.ac.cn)
[6,7]. Energy storage in traditional supercapacitors and rechargeable batteries is usually based on active solid electrode materials which limit energy density. If active materials with reversible redox properties can be stored in solution, high energy devices may be obtainable. The Li-Cu battery is an example of this, where a $\mathrm{Cu}$-cathode in aqueous electrolyte and a Li-anode in non-aqueous electrolyte, are separated by a lithium super-ionic conductor glass film (LISICON) in the hybrid electrolyte [8]. The redox flow battery (RFB) has recently also received much attention because of its high-efficiency, low cost, large-scale energy storage where active materials are dissolved in electrolyte solution. The vanadium redox flow battery is thought to be the most practical candidate [9]. Present RFBs are based on inorganic active materials restricted by limited mineral resources. Organic active materials are good candidates for energy storage devices, because they can be synthesized from biomass 
via green chemistry and are more easily recyclable [10]. The 2,2,6,6-tetramethyl-1-piperidinyloxy (TEMPO) nitroxide radical is a stable organic species which undergoes reversible reduction and oxidation. Poly(2,2,6,6-tetramethylpoperidinyloxy methacrylate) (PTMA) containing embedded TEMPO has been applied to both cathodic active materials in rechargeable batteries and positive electrode materials in hybrid electrochemical capacitors [11]. In the current study, we report a hybrid electrochemical energy storage device using multi-walled carbon nanotubes (MWCNTs) as the supporting electrode, where all active materials dissolved in aqueous solution. An important point is the use of the TEMPO nitroxide radical as the anodic active material and hydroquinone (HQ) as the cathodic active material, which were separated by an $\mathrm{OH}^{-}$-selective membrane. The devices electrochemical performance was investigated by cyclic voltammetry and galvanostatic charge-discharge testing. The device afforded high capacitance through nonfaradic reactions of supporting electrode via charge separation on the electrode material/electrolyte interfaces, and the faradic redox-reactions of TEMPO nitroxide radical between its different oxidation states [12]. While the former provides the well-known double layer capacitance, the latter results in high pseudocapacitance.

\section{Materials and methods}

The hybrid electrochemical energy storage device contained an anode and cathode separated by an $\mathrm{OH}^{-}$-selective membrane (Astom Corp, Japan.), as shown in Figure 1(a). The
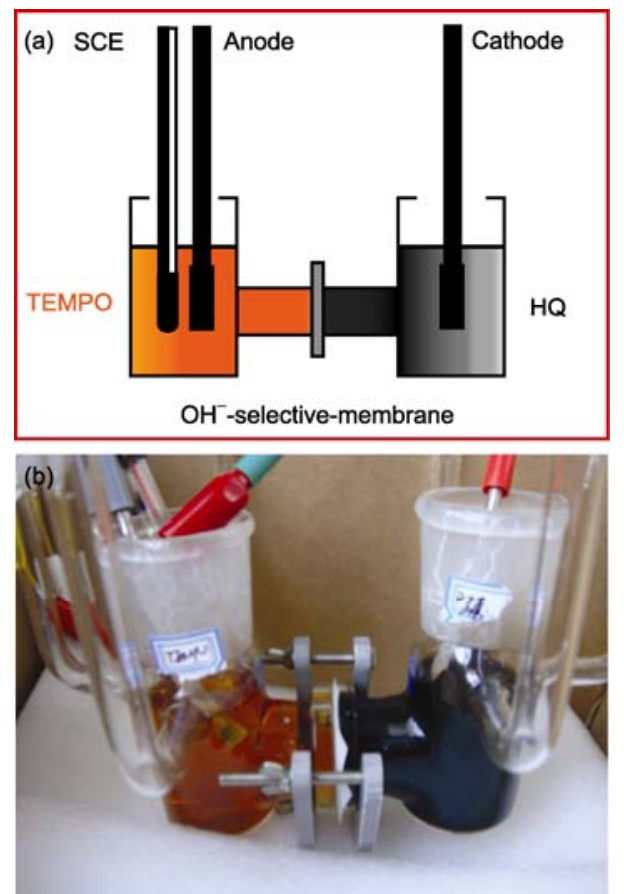

Figure 1 The hybrid electrochemical energy storage device prepared in this study: (a) schematic and (b) photograph. membrane permitted solely $\mathrm{OH}^{-}$to permeate it. A mixture of MWCNTs, carbon black and Teflonized acetylene black (TAB) at a weight ratio of $80: 10: 10$, was pasted onto a $\mathrm{Ni}$ net $(4 \mathrm{~cm} \times 0.8 \mathrm{~cm} \times 0.1 \mathrm{~mm})$ which formed the two supporting electrodes. The active powder $(\sim 1.5 \mathrm{mg})$ was pressed onto the Ni net and formed a pellet with $0.6 \mathrm{~cm} \times$ $0.6 \mathrm{~cm} \times 0.1 \mathrm{~mm}$ in length $\times$ width $\times$ thickness. Equimolar ratios of TEMPO nitroxide radical and HQ were dissolved in $100 \mathrm{~mL}$ of aqueous $1 \mathrm{~mol} / \mathrm{L} \mathrm{NaOH}$ as the active materials for the anode and cathode, respectively. Figure 1(a) shows that both the anode (working electrode) and reference electrode (saturated calomel electrode, SCE) were immersed in TEMPO/NaOH solution, while the cathode (counter electrode) was so in $\mathrm{HQ} / \mathrm{NaOH}$ solution. Figure 1(b) shows an optical image of a real device built in this study.

Cyclic voltammograms (CVs) were measured on a Parstat 2273 advanced electrochemical system, at scan rates varying from 1 to $100 \mathrm{mV} \mathrm{s}^{-1}$. Galvanostatic charge-discharge tests of the assembled device were carried out on an Arbin BT2000 system, over the voltage range $-0.8-0 \mathrm{~V}$ with respect to SCE under different current densities. All potentials reported were referenced to SCE.

\section{Results and discussion}

Figure 2(a) shows CV curves of the MWCNT electrode (line i), $5 \mathrm{mmol} / \mathrm{L}$ TEMPO nitroxide radical (line ii) and $5 \mathrm{mmol} / \mathrm{L} \mathrm{HQ}$ (line iii), in $1 \mathrm{~mol} / \mathrm{L} \mathrm{NaOH}$ solution at a scan rate of $10 \mathrm{mV} \mathrm{s}^{-1}$ and $-0.8-0 \mathrm{~V}$ voltage window. The CV curve for MWCNT exhibited a nearly rectangular shape without obvious peaks, which is typical for capacitive behavior. The $\mathrm{CV}$ curve for TEMPO nitroxide radical exhibited a reversible redox peak at $-0.4 \mathrm{~V}$ in $\mathrm{NaOH}$ solution, which was ascribed to the transition between the aminoxy anion state and the nitroxide radical state (Figure 3). This may have resulted in the redox capacitance. The difference between anodic $\left(E_{\mathrm{a}}:-393 \mathrm{mV}\right)$ and cathodic $\left(E_{\mathrm{c}}:-436 \mathrm{mV}\right)$ peak potentials was as small as $43 \mathrm{mV}$ at a $10-\mathrm{mV} \mathrm{s}^{-1}$ scan rate, which implied a fast reversible electrochemical reaction for the TEMPO nitroxide radical. The $\mathrm{CV}$ curve for $\mathrm{HQ}$ exhibited two redox pairs at around -0.6 and $-0.18 \mathrm{~V}$, the former $\left(E_{\mathrm{a}}:-633 \mathrm{mV} ; E_{\mathrm{c}}:-604 \mathrm{mV}\right)$ reflecting the reversible inter-conversion of $\mathrm{HQ}$ and 1,4-benzoquinone $\left(E_{\mathrm{c}}-E_{\mathrm{a}}=\right.$ $29 \mathrm{mV}$ ) (Figure 3). The peak current of the redox peak at $-0.18 \mathrm{~V}$ decreased with increasing scan rate (not shown), which might not be used for supercapacitors due to the irreversible redox reaction. The devices open circuit voltage was $-0.16 \mathrm{~V}$, and the initial cathodic peak represented the reduction of the TEMPO nitroxide radical to the aminoxy anion. HQ was correspondingly oxidized to 1,4-benzoquinone in aqueous $\mathrm{NaOH}$. Cycling tests in Figure 2(b) showed noobvious peak current loss after 90 cycles in $10 \mathrm{mmol} / \mathrm{L}$ TEMPO nitroxide radical, further demonstrating the excellent 

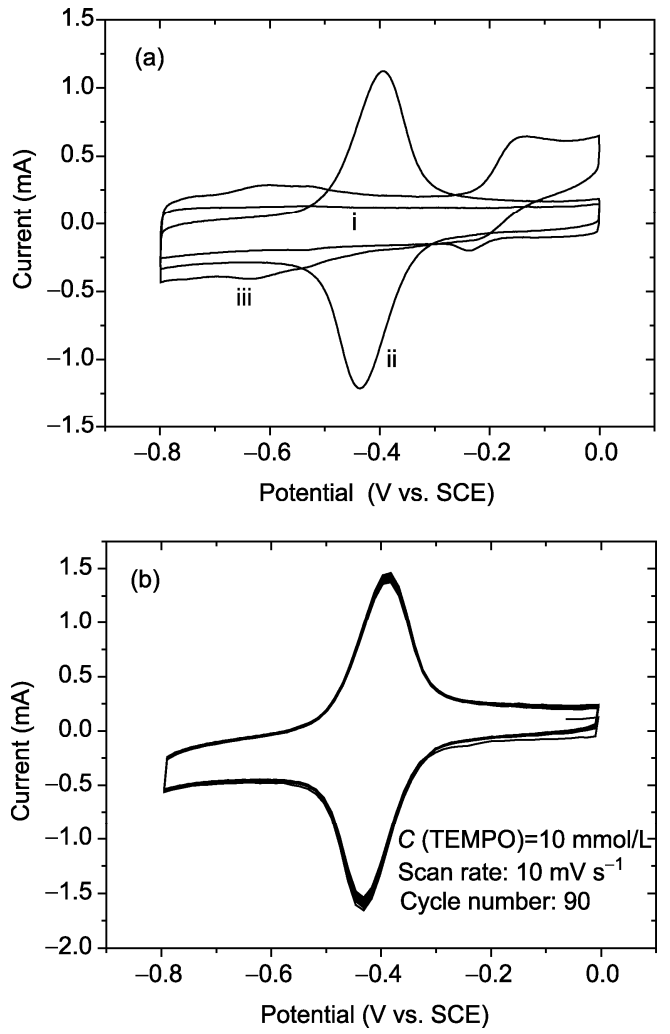

Figure 2 (a) $\mathrm{CV}$ curves of the MWCNT electrode in $0 \mathrm{mmol} / \mathrm{L}$ TEMPO nitroxide radical (i), $5 \mathrm{mmol} / \mathrm{L}$ TEMPO (ii) and $5 \mathrm{mmol} / \mathrm{L} \mathrm{HQ}$ (iii), in $1 \mathrm{~mol} / \mathrm{L}$ $\mathrm{NaOH}$ solution with a $10 \mathrm{mV} \mathrm{s}^{-1}$ scan rate. (b) CV curves in $10 \mathrm{mmol} / \mathrm{L}$ TEMPO nitroxide radical with a $10 \mathrm{mV} \mathrm{s}^{-1}$ scan rate.<smiles>C=CC#CC1(C)CC(O)CC(C)(C)N1[O-]</smiles>

Aminoxy anion<smiles>Oc1ccc(O)cc1</smiles>

$\mathrm{HQ}$ (Hydroquinone)<smiles>CC1(C)CC(O)CC(C)(C)N1O</smiles>

TEMPO<smiles>O=C1C=CC(=O)C=C1</smiles>

1,4-benzoquinone

Figure 3 Reversible redox reactions of TEMPO nitroxide radical and HQ.

chemical stability of this redox system.

The specific capacitance was calculated from the $\mathrm{CV}$ curves using the equation $C=Q / m V$, where $Q$ is the integration of current and time, $V$ is the voltage range, and $m$ is the mass of the MWCNT electrode. Specific capacitances for $1 \mathrm{~mol} / \mathrm{L} \mathrm{NaOH}$ electrolyte containing TEMPO nitroxide radicals at different concentrations are summarized in Figure 4 (a). Scan rates of 1, 2, 5, 10, 20, 50 and $100 \mathrm{mV} \mathrm{s}^{-1}$
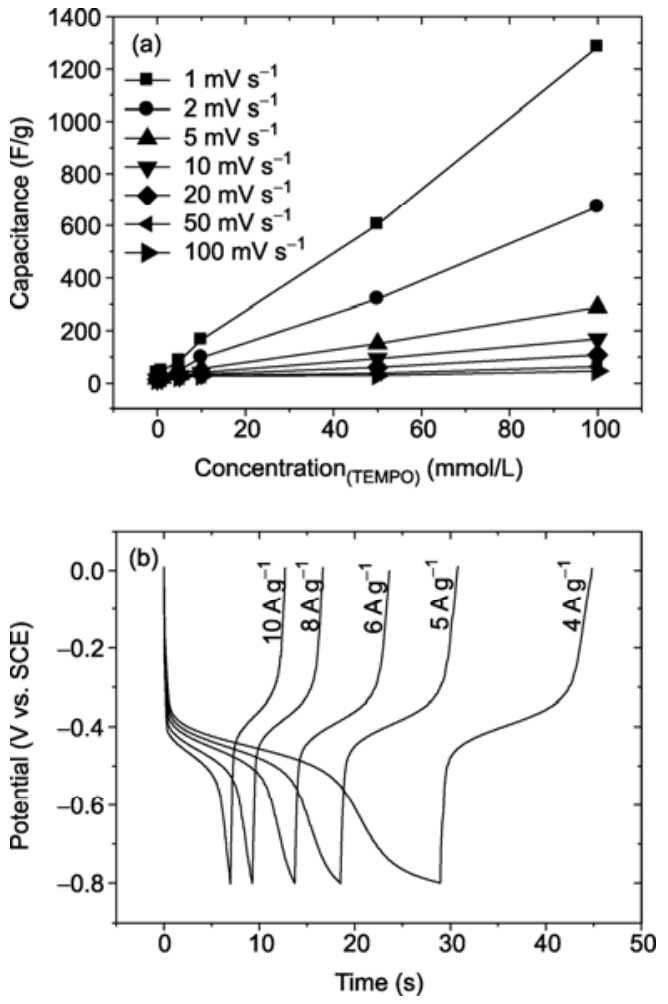

Figure 4 (a) Specific capacitance of the MWCNT electrode with increasing TEMPO nitroxide radical concentration, in $1 \mathrm{~mol} / \mathrm{L} \mathrm{NaOH}$ electrolyte at different scan rates. (b) Galvanostatic charging-discharging curves in $100 \mathrm{mmol} / \mathrm{L}$ TEMPO nitroxide radical at different current densities of $10,8,6,5$ and $4 \mathrm{~A} \mathrm{~g}^{-1}$.

were performed for each TEMPO nitroxide radical concentration. Figure 4(a) indicates that specific capacitance increased with increasing radical concentration and decreased with increasing scan rate. A greater quantity of TEMPO nitroxide radicals undergoing redox reaction may have been responsible for the former trend. The latter may have been attributed to less efficient diffusion of TEMPO nitroxide radicals to the MWCNT electrode reaction interface [13]. For a TEMPO nitroxide radical concentration of $100 \mathrm{mmol} / \mathrm{L}$, capacitance exceeded $1200 \mathrm{Fg}^{-1}$ when it was only $38 \mathrm{Fg}^{-1}$ in the absence of TEMPO nitroxide radical at a $1-\mathrm{mV} \mathrm{s}^{-1}$ scan rate. Thus, high pseudo-capacitance was contributed to by the TEMPO nitroxide radical redox reaction in aqueous $\mathrm{NaOH}$.

Galvanostatic charge/discharge measurements were carried out to further investigate the electrochemical performance of the device. A potential range $-0.8-0 \mathrm{~V}$ at different current densities were used for the tests, which were carried out in $100 \mathrm{mmol} / \mathrm{L}$ TEMPO nitroxide radical and $1 \mathrm{~mol} / \mathrm{L}$ $\mathrm{NaOH}$ solution. Figure 4 (b) shows the measurement was conducted by first discharging and then charging the device. Curves were comprised of three distinct parts: two sharp voltage drops from 0 to $-0.3 \mathrm{~V}$ and from -0.6 to $-0.8 \mathrm{~V}$, and one voltage plateau from -0.3 to $-0.6 \mathrm{~V}$. The two voltage drops may have been ascribed to the electric double- 
layer capacitance of the MWCNTs electrode, while the long plateau may have been caused by the combination of the electric double-layer capacitance from MWCNTs and the faradaic capacitance from the TEMPO nitroxide radical [14]. There was a discharging-charging voltage platform at $-0.4 \mathrm{~V}$, which was consistent with the redox peak at $-0.4 \mathrm{~V}$ observed for TEMPO nitroxide radical reaction. Discharge time increased with decreasing current density, hence capacitance increased. These results are in good agreement with those from the above $\mathrm{CV}$ curves.

\section{Conclusions}

A novel environmental-friendly hybrid energy storage device has been developed, with the TEMPO nitroxide radical serving as the active material, $\mathrm{HQ}$ as the counter active material and an $\mathrm{OH}^{-}$-selective separating membrane. The reversible redox reaction of the TEMPO nitroxide radical provided a significant pseudo-capacitance far beyond the electric double-layer capacitance of MWCNTs. The specific capacitance increased with increasing TEMPO nitroxide radical concentration. A maximum specific capacitance of $1200 \mathrm{~F} \mathrm{~g}^{-1}$ was obtained at the scan rate of $1 \mathrm{mV} \mathrm{s}^{-1}$. The electrode material and active material dissolved in solution could potentially be exchanged for alternative materials, with either high electric double-layer capacitance or high pseudo-capacitance within the appropriate electrochemical potential ranges. This simple design provides a new approach for fabricating high performance supercapacitorbattery hybrid energy storage devices.

This work was supported by the National Natural Science Foundation of
China (50730005 and 20821003), the National Key Project on Basic Research (2011CB935700 and 2009CB930400), and the Knowledge Innovation Program of the Chinese Academy of Sciences (KJCX2-YW-W26).

1 Arico A S, Bruce P, Scrosati B, et al. Nanostructured materials for advanced energy conversion and storage devices. Nat Mater, 2005, 4: 366-377

2 Armand M, Tarascon J M. Building better batteries. Nature, 2008, 451: 652-657

3 Guo Y G, Hu J S, Wan L J. Nanostructured materials for electrochemical energy conversion and storage devices. Adv Mater, 2008, 20: $2878-2887$

4 Li H, Wang Z X, Chen L Q, et al. Research on advanced materials for Li-ion batteries. Adv Mater, 2009, 21: 4593-4607

5 Yan J, Wei T, Fan Z, et al. Preparation of graphene nanosheet/carbon nanotube/polyaniline composite as electrode material for supercapacitors. J Power Sources, 2010, 195: 3041-3045

6 Li H Q, Wang Y G, Na H T, et al. Rechargeable Ni-Li battery integrated aqueous/nonaqueous system. J Am Chem Soc, 2009, 131: 15098-15099

7 Li H Q, Wang Y G, He P, et al. A novel rechargeable Li-AgO battery with hybrid electrolytes. Chem Comm, 2010, 46: 2055-2057

8 Wang Y G, Zhou H S. A new type rechargeable lithium battery based on a Cu-cathode. Electrochem Commun, 2009, 11: 1834-1837

9 Skyllas-Kazacos M, Kazacos G, Poon G, et al. Recent advances with UNSW vanadium-based redox flow batteries. Int J Energy Res, 2010, 34: $182-189$

10 Liao C, Wei Z D, Chen S G, et al. Synergistic effect of polyanilinemodified $\mathrm{Pd} / \mathrm{C}$ catalysts on formic acid oxidation in a weak acid medium (NH)SO. J Phys Chem C, 2009, 113: 5705-5710

11 Li H Q, Zou Y, Xia Y Y. A study of nitroxide polyradical/activated carbon composite as the positive electrode material for electrochemical hybrid capacitor. Electrochimi Acta, 2007, 52: 2153-2157

12 Nishide $\mathrm{H}$, Iwasa $\mathrm{S}, \mathrm{Pu}$ Y J, et al. Organic radical battery: Nitroxide polymers as a cathode-active material. Electrochimi Acta, 2004, 50: 827-831

13 Maier J. Nanoionics: Ion transport and electrochemical storage in confined systems. Nat Mater, 2005, 4: 805-815

14 Yan J, Wei T, Shao B, et al. Preparation of a graphene nanosheet/ polyaniline composite with high specific capacitance. Carbon, 2010, 48: 487-493

Open Access This article is distributed under the terms of the Creative Commons Attribution License which permits any use, distribution, and reproduction in any medium, provided the original author(s) and source are credited. 